MATHEMATICS OF COMPUTATION

Volume 71, Number 237, Pages 49-76

S $0025-5718(01) 01346-1$

Article electronically published on October 4, 2001

\title{
A POSTERIORI ERROR ESTIMATES FOR GENERAL NUMERICAL METHODS FOR HAMILTON-JACOBI EQUATIONS. PART I: THE STEADY STATE CASE
}

\author{
SAMUEL ALBERT, BERNARDO COCKBURN, DONALD A. FRENCH, \\ AND TODD E. PETERSON
}

\begin{abstract}
A new upper bound is provided for the $\mathrm{L}^{\infty}$-norm of the difference between the viscosity solution of a model steady state Hamilton-Jacobi equation, $u$, and any given approximation, $v$. This upper bound is independent of the method used to compute the approximation $v$; it depends solely on the values that the residual takes on a subset of the domain which can be easily computed in terms of $v$. Numerical experiments investigating the sharpness of the a posteriori error estimate are given.
\end{abstract}

\section{INTRODUCTION}

This paper is the first of a series devoted to the study of a posteriori error estimates for Hamilton-Jacobi equations. The Hamilton-Jacobi equations arise in several areas of applications, such as evolving interfaces in geometry, fluid mechanics, computer vision, and materials science (see Sethian [16]); the shape-from-shading problem (see, for instance, Lions, Rouy, and Tourin [13]); and optimization, control, and differential games (see the references in Crandall and Lions [7]). Because of these many applications, there is an interest in finding algorithms that produce numerical approximations with a guaranteed precision set beforehand by the practitioner. Thus it is important to be able to estimate the quality of any given approximation $v$ solely in terms of computable quantities; this is what a posteriori error estimates provide.

In this paper, we show how to obtain new a posteriori error estimates for Hamilton-Jacobi equations and perform analytical and numerical experiments to study their sharpness. To render the presentation of the ideas as clear as possible, we consider the simple setting of periodic viscosity solutions of the following model steady state Hamilton-Jacobi equation:

$$
u+H(\nabla u)=f \quad \text { in } \mathbb{R}^{d},
$$

Received by the editor April 10, 1997 and, in revised form, April 17, 2000.

2000 Mathematics Subject Classification. Primary 54C40, 14E20; Secondary 46E25, 20C20.

Key words and phrases. Error estimates, Hamilton-Jacobi.

The second author was partially supported by the National Science Foundation (Grant DMS9807491) and by the University of Minnesota Supercomputer Institute.

The third author was partially supported by the Taft Foundation through the University of Cincinnati.

(C)2001 American Mathematical Society 
where $u$ and $f$ are periodic in each coordinate with period 1. Extensions to the transient case and to second-order equations will be treated in Parts II and III, respectively, of this series.

Before describing our result, let us stress the fact that one of the main current trends in the numerical analysis of partial differential equations is the development of a posteriori error estimates. The more traditional error estimates, nowadays called a priori because they can be obtained prior to the computation of the approximate solution, have been (and still are) the main tool of theoretical analysis in this field for the past few decades. However, for practical applications they are not very useful because they depend on the numerical method used to compute the approximate solution $v$, involve information that is not known about the exact solution (which can only be crudely estimated), and cannot capture the features of the particular problem under consideration. These difficulties prompted the idea of developing error estimates that depend solely on the approximate solution; as a consequence, they can only be used after one has computed the approximate solution, that is, a posteriori. These estimates are nothing but continuous dependence results for the equation under consideration; this is why, unlike the a priori error estimates, they do not depend on the numerical method used to compute the approximation $v$. For example, to get an a posteriori error estimate in our case, the idea is to write

$$
v+H(\nabla v)=g,
$$

and then try to obtain a continuous dependence result of the form

$$
\|u-v\| \leq \Psi(f-g)=\Psi(-R(v)),
$$

where $R(v)=v+H(\nabla v)-f$ is the so-called residual of $v$. In order to evaluate the functional $\Psi$, it is usually necessary to obtain a priori estimates of approximations to the solution of the so-called adjoint problem; see the book by Eriksson, Estep, Hansbo and Johnson [8] for an introduction to the subject. However, for a few equations and norms $\|\cdot\|$, the functional $\Psi$ can be evaluated without having to solve an adjoint problem; this is precisely our case.

The error estimate we obtain on the approximate solution $v$ is of the form

$$
\|u-v\|_{L^{\infty}(\Omega)} \leq \Phi(v),
$$

where the nonlinear functional $\Phi$ depends on the Hamiltonian $H$, the right hand side $f$, and the domain $\Omega$, but is totally independent of the numerical method used to compute $v$. The error estimate (1.2) can thus be applied if $v$ is obtained by means of a finite difference scheme like the ENO scheme developed by Osher and Shu [14, by means of a finite element method like the discontinuous Galerkin (DG) method of $\mathrm{Hu}$ and Shu 10] or the Petrov-Galerkin method used by Barth and Sethian 2, a finite volume method like the intrinsic monotone scheme of Abgrall [1] or the ones devised by Kossioris, Makridakis and Souganidis [11].

As expected, the functional $\Phi$ depends on the residual of $v$. However, a novel feature of the a posteriori error estimate (1.2) is that only the values that the residual takes on a suitably defined subset of the domain $\Omega$ are used to evaluate $\Phi$. This subset, which can be chosen in terms of $v$ only, does not contain neighborhoods of points at which $v$ has kinks (discontinuities in first derivatives) or at which it has a large Hessian. This dramatically enhances the sharpness of the a posteriori error estimate, since these are the only points at which the residual might remain 
of order one, even for very good approximate solutions $v$; see the analytic examples of Section 3 and the numerical experiments of Section 4.

To obtain the error estimate (1.2), we use the elegant technique introduced by Crandall, Evans and Lions [5] to study viscosity solutions of Hamilton-Jacobi equations. This technique can deal with arbitrary Hamiltonians, works in the same way regardless of the space dimension, and leads naturally to the use of the $\mathrm{L}^{\infty}$ norm (which is why we use this norm). The use of any other norm involves the resolution of the so-called adjoint equation. Indeed, Lin and Tadmor [12] have recently obtained error estimates in the $\mathrm{L}^{1}$-norm and, not surprisingly, had to consider the resolution of an adjoint equation. Since the study of its solution was successfully carried out only for strictly convex Hamiltonians, their error estimate holds only in that case.

Several authors have obtained $\mathrm{L}^{\infty}$-error estimates for Hamilton-Jacobi equations, but our result is different in many respects. Crandall and Lions [5] obtained an a priori error estimate between the viscosity solution and the approximation $v$ given by a monotone scheme defined in Cartesian grids. In our setting, their result reads as follows:

$$
\|u-v\|_{\mathrm{L}^{\infty}(\Omega)} \leq C(\Delta x)^{1 / 2},
$$

where $\Delta x$ is the maximum mesh size. This result holds for viscosity solutions that might displays kinks. Souganidis [17] extended this estimate to more general Hamiltonians and to general finite difference schemes in Cartesian grids. Recently, Abgrall [1] introduced the intrinsic monotone schemes for unstructured meshes and proved that the same error estimate holds. Perthame and Sanders [15] proved an error estimate of the same flavor for the Neumann problem for a nonlinear parabolic singular perturbation. Falcone and Ferretti [9] obtained a priori error estimates, assuming the viscosity solution of the Hamilton-Jacobi-Bellman equation to be very smooth. Their schemes were constructed by using the discrete dynamic programming principle and were devised to converge fast to the solution; their results apply to convex Hamiltonians $H$. All these a priori error estimates are not useful in practical applications for the reasons described at the beginning of this section. In contrast, the estimate (1.2) is an a posteriori error estimate that holds regardless of how $v$ was computed, is independent of the smoothness of the exact solution and takes into account the particularities of the specific problem (as our numerical results show).

Note also that whereas the main objective of all the above mentioned error estimates is to obtain a rate of convergence of the approximate solution, our main objective is to be able to obtain, for any given approximate solution $v$, an accurate upper bound for the value $\|u-v\|_{L^{\infty}(\Omega)}$ that solely depends on $v$. To quantify the accuracy of the bound, we introduce the so-called effectivity index

$$
\operatorname{ei}(u, v)=\Phi(v) /\|u-v\|_{L^{\infty}(\Omega)},
$$

and study how close it is to the ideal value of 1 .

A preliminary numerical study of the corresponding quantity in the framework of one-dimensional time-dependent nonlinear scalar conservation laws was performed by Cockburn and Gau [4]; the approximate solution they used was computed by using the monotone scheme of Engquist and Osher. Their results give a ratio that 
is very close to 1 for nonlinear convection with a smooth solution and for linear convection regardless of the smoothness of the exact solution. Since the integral of the entropy solution of a one-dimensional conservation law is nothing but the viscosity solution of a Hamilton-Jacobi equation, it is reasonable to expect a similar outcome for Hamilton-Jacobi equations; in this paper, we show that small effectivity indices are indeed obtained. For the difficult case of nonlinear Hamiltonians with viscosity solutions that display kinks, our numerical experiments show that the effectivity index ei $(u, v)$ is proportional to $|\ln \Delta x|$ for monotone schemes. This is to be contrasted with the effectivity index of order $(\Delta x)^{-1 / 2}$ that would have been obtained had we used the a priori Crandall and Lions [7] estimate, and with the effectivity index of order $(\Delta x)^{-1}$ that would have been obtained had we used the a posteriori estimate of Cockburn and Gau 4 for the corresponding scalar hyperbolic conservation law.

We also study the effectivity index for the modern high-resolution DG method proposed by $\mathrm{Hu}$ and Shu [10]. A straightforward application of our a posteriori error estimate produces effectivity indices proportional to $(\Delta x)^{-1}$ when the exact solution is very smooth; this is due to the fact that these high-order accurate DG methods produce a highly oscillatory residual, as is typical of Galerkin methods. However, we can obtain another approximate solution by using a simple post-processing (that maintains the order of accuracy of the approximation) for which the effectivity index remains constant, and reasonably small, as the mesh size decreases. In the case of viscosity solutions displaying kinks, the results for the DG methods of $\mathrm{Hu}$ and Shu [10] are similar to the ones given by monotone schemes. This is due to the fact that in such a case, the maximum error occurs at the kinks and it is precisely around those that the DG method employs a low degree polynomial approximation.

The important issue of adaptivity, that is, the issue of how to compute an approximate solution $v$ satisfying

$$
\Phi(v) \leq \tau,
$$

for any given tolerance $\tau$, with minimal computational effort will be addressed elsewhere. This is a difficult problem that requires study of how the functional $\Phi(v)$ depends on the grid and the numerical scheme used to compute $v$; it falls beyond the scope of this paper. However, our numerical experiments discussed in Section 4 do give some insight into the use of the a posteriori error estimate for adaptivity purposes.

This paper is organized as follows. In Section 2, we state, discuss, and give a proof of the a posteriori error estimate. In Section 3, we illustrate the application of the estimate (1.2) and assess its sharpness; we study three important cases that can be considered as prototypical and perform all the computations by hand. These results give us indications of how to choose several parameters relevant to the proper and efficient evaluation of the a posteriori error estimate as applied to numerical schemes. We devote Section 4 to the numerical study of the effectivity index ei $(u, v)$ where $v$ is the approximate solution provided either by a monotone scheme or by the DG method of $\mathrm{Hu}$ and Shu [10]; most of the numerical experiments are done in a one-dimensional setting, but we also present a couple of two-dimensional test problems. Finally, we end in Section 5 with some extensions and concluding remarks. 


\section{THE A POSTERIORI ERROR ESTIMATE}

2.1. Viscosity solutions. We start this subsection by recalling the definition of viscosity solutions, and some of their basic properties. We use the definition introduced by Crandall, Evans and Lions [5]; this is not the original definition of Crandall and Lions [ [6] but it is equivalent to it and it is convenient for our purposes.

To state the definition, we need the notion of semidifferentials of a function. The superdifferential of a function $u$ at a point $x, D^{+} u(x)$, is the set of all vectors $p$ in $\mathbb{R}^{d}$ such that

$$
\limsup _{y \rightarrow x}(u(y)-\{u(x)+(y-x) \cdot p\}) \leq 0,
$$

and the subdifferential of a $u$ at a point $x, D^{-} u(x)$, is the set of all vectors $p$ in $\mathbb{R}^{d}$ such that

$$
\liminf _{y \rightarrow x}(u(y)-\{u(x)+(y-x) \cdot p\}) \geq 0 .
$$

Below we will use some elementary properties of these semidifferentials, often without comment.

We also need to define the following quantity:

$$
R(u ; x, p)=u(x)+H(p)-f(x),
$$

which is just the residual of $u$ at $x$ if $p=\nabla u(x)$. For this reason, we also call $R$ the residual; notice, however, that the residual of $u, R(u ; \cdot, \cdot)$, has two variables and not just one.

We are now ready to define the viscosity solution of (1.1).

Definition 2.1. [5] A viscosity solution of the Hamilton-Jacobi equation (1.1) is a continuous periodic function on $\mathbb{R}^{d}$ such that, for all $x$ in $\mathbb{R}^{d}$,

$$
+R(u ; x, p) \leq 0 \quad \forall p \in D^{+} u(x), \quad \text { and } \quad-R(u ; x, p) \leq 0 \quad \forall p \in D^{-} u(x) .
$$

Note that this definition can be written more compactly as

$$
\sigma R(u ; x, p) \leq 0, \quad \forall p \in D^{\sigma} u(x), \quad \sigma \in\{+,-\} .
$$

This $\sigma$ notation will be useful below.

2.2. $\mathrm{L}^{\infty}$-contraction property. The viscosity solution $u$ of (1.1) may be compared with the viscosity solution of the equation

$$
v+H(\nabla v)=g,
$$

via the so-called $\mathrm{L}^{\infty}$-contraction property, see Theorem 2.1 in [5], namely,

$$
\|u-v\|_{\mathrm{L}^{\infty}} \leq\|f-g\|_{\mathrm{L}^{\infty}}
$$

(see also Theorem II.1 in Crandall and Lions [6]). From this inequality, it is very easy to obtain an a posteriori error estimate if $v$ is any continuous periodic function on $\mathbb{R}^{d}$. Indeed, let us define $g$ by

$$
g(x)= \begin{cases}\sup \left\{v(x)+H(p): p \in D^{+} v(x)\right\}, & \text { if } D^{+} v(x) \neq \emptyset, \\ \inf \left\{v(x)+H(p): p \in D^{-} v(x)\right\}, & \text { if } D^{-} v(x) \neq \emptyset .\end{cases}
$$


Note that if $D^{+} v(x)$ and $D^{-} v(x)$ are both nonempty at some point $x$, then $v$ is differentiable at $x$, and $D^{+} v(x)=D^{-} v(x)=\{\nabla v(x)\}$, so $g(x)$ is well defined. With $g$ so defined, $v$ is the viscosity solution of $v+H(\nabla v)=g$ and, by (2.2) ,

$$
\|u-v\|_{\mathrm{L}^{\infty}} \leq \sup \left\{|R(v ; x, p)|: x \in \mathbb{R}^{d}, p \in D^{+} v(x) \cup D^{-} v(x)\right\} .
$$

This is in fact a very simple a posteriori error estimate of the form we seek. However, it turns out that this estimate is not always sharp; in some cases the quantity on the right of (2.3) remains of order one even as $v$ converges to $u$, see the second of the analytical examples in Section 3.

2.3. The a posteriori error estimate. Our main result gives an upper bound for the following seminorms:

$$
\begin{aligned}
& |u-v|_{-}=\sup _{x \in \Omega}(u(x)-v(x))^{+}, \\
& |u-v|_{+}=\sup _{x \in \Omega}(v(x)-u(x))^{+},
\end{aligned}
$$

where $w^{+} \equiv \max \{0, w\}$. To state our a posteriori error estimate, we need to introduce some notation. We start with the following quantity:

$$
\begin{aligned}
R_{\epsilon}(u ; x, p) & =u(x)+H(p)-f(x-\epsilon p)-\frac{1}{2} \epsilon|p|^{2} \\
& =R(u ; x, p)+f(x)-f(x-\epsilon p)-\frac{1}{2} \epsilon|p|^{2} .
\end{aligned}
$$

Since $R_{\epsilon}$ is nothing but the residual $R$ when $\epsilon=0$, and since the evaluation of $R_{\epsilon}$ involves the evaluation of $f$ at the shifted point $x-\epsilon p$, we call $R_{\epsilon}$ the shifted residual.

To characterize the subset of the domain $\mathbb{R}^{d} \times \mathbb{R}^{d}$ on which the shifted residual of $v$ will be evaluated, we use the paraboloid $P_{v}$ defined as follows:

$$
P_{v}(x, p, \kappa ; y)=v(x)+(y-x) \cdot p+\frac{\kappa}{2}|y-x|^{2}, \quad y \in \mathbb{R}^{d},
$$

where $x$ is a point in $\mathbb{R}^{d}, p$ is a vector of $\mathbb{R}^{d}$, and $\kappa$ is a real number. Note that in one space dimension, $P_{v}\left(x, v^{\prime}(x), v^{\prime \prime}(x) ; \cdot\right)$ is nothing but the Taylor polynomial of degree two of $v$ at $x$.

We are now ready to state the a posteriori error estimate.

Theorem 2.2 (A posteriori error estimate). Let $u$ be the viscosity solution of the equation (1.1) and let $v$ be any continuous function on $\mathbb{R}^{d}$ periodic in each coordinate with period 1 . Then, for $\sigma \in\{-,+\}$, we have that

$$
|u-v|_{\sigma} \leq \inf _{\epsilon \geq 0} \Phi_{\sigma}(v ; \epsilon)
$$

where

$$
\Phi_{\sigma}(v ; \epsilon)=\sup _{(x, p) \in \mathcal{A}_{\sigma}(v ; \epsilon)}\left(\sigma R_{\sigma \epsilon}(v ; x, p)\right)^{+} .
$$

The set $\mathcal{A}_{\sigma}(v ; \epsilon)$ is the set of elements $(x, p)$ satisfying

$$
\begin{aligned}
& x \in \mathbb{R}^{d}, \\
& p \in D^{\sigma} v(x), \\
& \sigma\left\{v(y)-P_{v}(x, p, \sigma / \epsilon ; y)\right\} \leq 0 \quad \forall y \in \mathbb{R}^{d}
\end{aligned}
$$

(for $\epsilon=0$, only the first two conditions apply). 


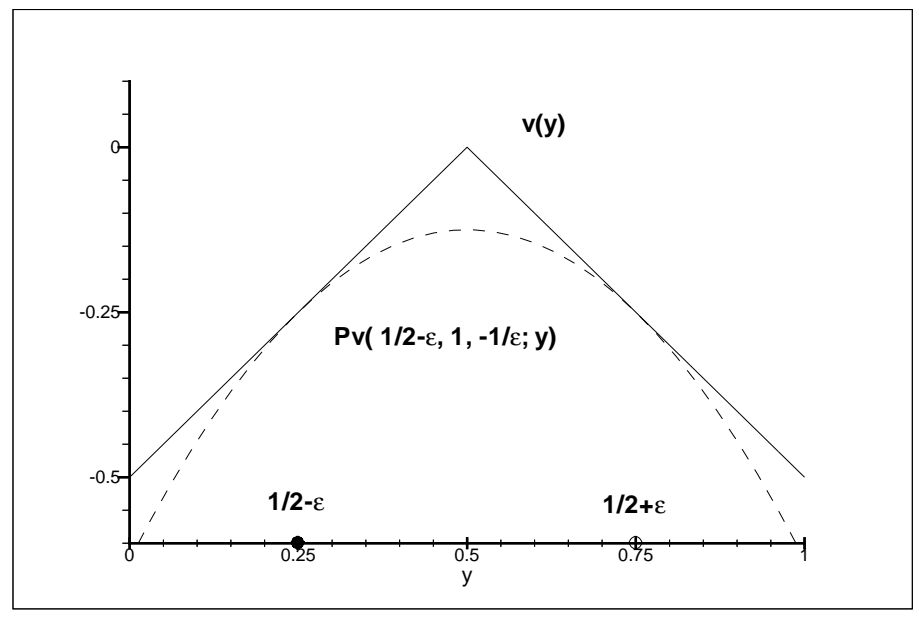

FiguRE 1. The parabola $y \mapsto P v(1 / 2-\epsilon, 1,-1 / \epsilon ; y)$ for $\epsilon=1 / 4$.

We want to stress several important points concerning this result.

a. The $\mathbf{L}^{\infty}$-error estimate. From the estimates of the seminorms $|u-v|_{\sigma}$, we obtain the desired error estimate (1.2), namely,

$$
\|u-v\|_{\mathrm{L}^{\infty}}=\max _{\sigma \in\{-,+\}}|u-v|_{\sigma} \leq \max _{\sigma \in\{-,+\}} \inf _{\epsilon \geq 0} \Phi_{\sigma}(v ; \epsilon) .
$$

b. The error estimate with $\epsilon=0$. Note that we can always set $\epsilon=0$ in the above inequality and recover the simple estimate (2.3); moreover, if $v$ is the viscosity solution of $v+H(\nabla v)=g$, we recover the $\mathrm{L}^{\infty}$ contraction property.

The estimate (2.3) is remarkable in its simplicity and provides a sharp upper bound for smooth viscosity solutions and approximate solutions with nonoscillatory residuals, as can be seen in the first analytic experiment of Section 3 and in some of the numerical experiments in Section 4; see also the numerical results for nonlinear conservation laws in [4. However, when the exact solution is not smooth, the Hamiltonian is nonlinear, and the numerical scheme is monotone, the upper bound given by the estimate (2.3) is of order one whereas the estimate (2.8) gives an upper bound of order $\Delta x|\ln \Delta x|$ only. In order to understand the mechanism responsible for this improvement, we need to illustrate the definition of the set $\mathcal{A}_{\sigma}(v ; \epsilon)$.

c. The set $\mathcal{A}_{\sigma}(v ; \epsilon)$. The definition of $\mathcal{A}_{\sigma}(v ; \epsilon)$ states that the paraboloid $P_{v}(x, p,-1 / \epsilon ; \cdot)$ must remain below the graph of $v$ for $\sigma=-$, and that the paraboloid $P_{v}(x, p,+1 / \epsilon ; \cdot)$ must remain above the graph of $v$ for $\sigma=+$ (see Figure11). To give an example of this set, consider $\mathcal{A}_{-}(v ; \epsilon)$ for the periodic continuous function $v(y)=-|y-1 / 2|$ defined on $\Omega=[0,1)$. A short calculation gives that

$$
\mathcal{A}_{-}(v ; \epsilon)= \begin{cases}\{0\} \times[-1,1] \cup(0,1 / 2-\epsilon] \times\{1\} \cup[1 / 2+\epsilon, 1) \times\{-1\}, & \text { if } \epsilon<1 / 2, \\ \{0\} \times[-1 / 2 \epsilon, 1 / 2 \epsilon], & \text { otherwise. }\end{cases}
$$

Note that $(x, p) \notin A_{-}(v ; \epsilon)$ if $x \in(1 / 2-\epsilon, 1 / 2+\epsilon)$; that is, a neighborhood of the kink of $v$, located at $x=1 / 2$, has been excluded. Also note that if $\epsilon$ is bigger that $\varepsilon$, then the set $A_{-}(v ; \epsilon)$ is included in $A_{-}(v ; \varepsilon)$. 
d. Smoothness of the mapping $\epsilon \mapsto \Phi_{\sigma}(v ; \epsilon)$. When $f$ and the approximate solution $v$ are Lipschitz, the function $\Phi_{\sigma}(v ; \epsilon)$ cannot be bigger than $\Phi_{\sigma}(v ; \varepsilon)$, if $\epsilon>\varepsilon$, by a quantity exceeding $C(\epsilon-\varepsilon)$, where

$$
C=\|v\|_{\operatorname{Lip}(\Omega)}\left(\frac{1}{2}\|v\|_{\operatorname{Lip}(\Omega)}+\|f\|_{\operatorname{Lip}(\Omega)}\right) .
$$

To see this, consider the following computations:

$$
\begin{aligned}
\Phi_{\sigma}(v ; \epsilon) & =\sup _{(x, p) \in \mathcal{A}_{\sigma}(v ; \epsilon)}\left(\sigma R_{\sigma \epsilon}(v ; x, p)\right)^{+} \\
& \leq \sup _{(x, p) \in \mathcal{A}_{\sigma}(v ; \varepsilon)}\left(\sigma R_{\sigma \epsilon}(v ; x, p)\right)^{+} \quad \text { since } \mathcal{A}_{\sigma}(v ; \epsilon) \subset \mathcal{A}_{\sigma}(v ; \varepsilon), \\
& \leq \sup _{(x, p) \in \mathcal{A}_{\sigma}(v ; \varepsilon)}\left(\left(\sigma R_{\sigma \varepsilon}(v ; x, p)\right)^{+}+\left(\sigma R_{\sigma \epsilon}(v ; x, p)-\sigma R_{\sigma \varepsilon}(v ; x, p)\right)^{+}\right) \\
& \leq \Phi_{\sigma}(v ; \varepsilon)+\sup _{(x, p) \in \mathcal{A}_{\sigma}(v ; \varepsilon)}\left(\sigma R_{\sigma \epsilon}(v ; x, p)-\sigma R_{\sigma \varepsilon}(v ; x, p)\right)^{+},
\end{aligned}
$$

and since

$$
\begin{aligned}
& R_{\sigma \epsilon}(v ; x, p)-R_{\sigma \varepsilon}(v ; x, p) \\
& \quad=-f(x-\sigma \epsilon p)+f(x-\sigma \varepsilon p)-\frac{\sigma}{2}(\epsilon-\varepsilon)|p|^{2}
\end{aligned}
$$

we get

$$
\Phi_{\sigma}(v ; \epsilon) \leq \Phi_{\sigma}(v ; \varepsilon)+C(\epsilon-\varepsilon)
$$

as claimed. In other words, $\Phi_{\sigma}(v ; \cdot) \in \operatorname{Lip}^{+}\left(\mathbb{R}^{+}\right)$.

e. The search for the optimal value of $\epsilon$. What allows the error estimate (2.8) to achieve bounds lower than (2.3) is the possibility of playing with the parameter $\epsilon$. As we just saw, the size of the set $\mathcal{A}_{\sigma}(v ; \epsilon)$ decreases as the auxiliary parameter $\epsilon$ increases; this induces a tendency for the upper bound to decrease as $\epsilon$ is increased. On the other hand, as $\epsilon$ increases, the signed shifted residual $\sigma R_{\sigma \epsilon}$ might also increase. The optimal value of $\epsilon$ is obtained by balancing these two tendencies.

f. The paraboloid test. To compare $v$ to the paraboloid $P_{v}$ in order to evaluate the condition (2.8), which we call the paraboloid test, could be very expensive computationally even if one takes advantage of the periodicity of the functions. In subsection 4.2 , we discuss a practical way to alleviate this when the function $v$ is Lipschitz.

2.4. Proof of the a posteriori error estimate. We prove the result for $\sigma=-$; the proof for the case $\sigma=+$ is similar. Set $\Omega=[0,1)^{d}$. Given $\epsilon>0$, define the auxiliary function

$$
\psi(x, y)=u(x)-v(y)-\frac{|x-y|^{2}}{2 \epsilon},
$$

and let $(\hat{x}, \hat{y}) \in \Omega \times \Omega$ be such that

$$
\psi(\hat{x}, \hat{y}) \geq \psi(x, y) \quad \forall x, y \in \Omega ;
$$

such a point exists since $\psi$ is continuous and periodic on $\Omega \times \Omega$. Set $\hat{p}=(\hat{x}-\hat{y}) / \epsilon$. 
We assume that $|u-v|_{-}>0$, otherwise there is nothing to prove. In this case, we have

$$
\begin{aligned}
|u-v|_{-}= & \sup _{x \in \Omega}\{u(x)-v(x)\} \\
= & \sup _{x \in \Omega} \psi(x, x) \\
\leq & \sup _{x, y \in \Omega} \psi(x, y) \\
= & \psi(\hat{x}, \hat{y})=u(\hat{x})-v(\hat{y})-\frac{|\hat{x}-\hat{y}|^{2}}{2 \epsilon} \\
= & {[u(\hat{x})+H(\hat{p})-f(\hat{x})] } \\
& -\left[v(\hat{y})+H(\hat{p})-f(\hat{y})+f(\hat{y})-f(\hat{x})+\frac{|\hat{x}-\hat{y}|^{2}}{2 \epsilon}\right] \\
= & R(u ; \hat{x}, \hat{p})-\left[R(v ; \hat{y}, \hat{p})+f(\hat{y})-f(\hat{x})+\frac{|\hat{x}-\hat{y}|^{2}}{2 \epsilon}\right] .
\end{aligned}
$$

Since the mapping $x \mapsto \psi(x, \hat{y})$ has a maximum at $x=\hat{x}$, we have that

$$
0 \in D_{x}^{+} \psi(\hat{x}, \hat{y})=D^{+} u(\hat{x})-\hat{p}
$$

and so

$$
\hat{p} \in D^{+} u(\hat{x})
$$

Since $u$ is the viscosity solution, this implies that $R(u ; \hat{x}, \hat{p}) \leq 0$, and hence

$$
\begin{aligned}
|u-v|_{-} & \leq-\left[R(v ; \hat{y}, \hat{p})+f(\hat{y})-f(\hat{y}+\epsilon \hat{p})+\frac{1}{2} \epsilon|\hat{p}|^{2}\right] \\
& =-R_{-\epsilon}(v ; \hat{y}, \hat{p}),
\end{aligned}
$$

where we have used the definition of $\hat{p}$ to write $\hat{x}=\hat{y}+\epsilon \hat{p}$.

Finally, since $\psi(\hat{x}, \hat{y}) \geq \psi(\hat{x}, y)$ for all $y \in \Omega$, we have that

$$
\begin{aligned}
v(y) & \geq v(\hat{y})+\frac{|\hat{x}-\hat{y}|^{2}}{2 \epsilon}-\frac{|\hat{x}-y|^{2}}{2 \epsilon} \\
& =v(\hat{y})+\hat{p} \cdot(y-\hat{y})-\frac{|y-\hat{y}|^{2}}{2 \epsilon},
\end{aligned}
$$

i.e., that $v(\cdot) \geq P v(\hat{y}, \hat{p},-1 / \epsilon ; \cdot)$; note that this implies that $\hat{p} \in D^{-} v(\hat{y})$. We thus have

$$
|u-v|_{-} \leq \sup \left\{-R_{-\epsilon}(v ; y, p): y \in \Omega, p \in D^{-} v(y), v(\cdot) \geq P v(y, p,-1 / \epsilon ; \cdot)\right\} .
$$

Since this inequality holds for any $\epsilon>0$ and since a similar result can be obtained for $\epsilon=0$, the desired result follows. This completes the proof of the a posteriori error estimate of Theorem 2.2 ,

\section{Analytical examples}

We will apply Theorem 2.2 to three different examples for which all computations can be done analytically, to obtain an idea of the sharpness of the error estimate.

a. Smooth $u$, nonoscillatory $R(v ; \cdot, \cdot)$. We take $H(p)=-\frac{1}{4 \pi^{2}} p^{2}$ and $f(x)=$ $\cos ^{4}(\pi x)$. The exact solution of (1.1) is then $u(x)=\cos ^{2}(\pi x)$, a smooth function. We take

$$
v(y)=c u(y)
$$


where $c \in(0,1)$; the residual $R(v ; \cdot, \cdot)$ is a smooth function that does not oscillate. We start by estimating $|u-v|_{-}$. By Theorem 2.2 we have

$$
\begin{aligned}
\left.|u-v|\right|_{-} & \leq \inf _{\epsilon \geq 0} \Phi_{-}(v ; \epsilon) \leq \Phi_{-}(v ; 0) \\
& =\sup _{(y, p) \in \mathcal{A}_{-}(v ; 0)}(-R(v ; y, p))^{+}=\sup _{y \in[0,1)}\left(-R\left(v ; y, v^{\prime}(y)\right)\right)^{+} \\
& =(1-c),
\end{aligned}
$$

which is the best possible estimate. To estimate $|u-v|_{+}$, we have, again by Theorem 2.2,

$$
\begin{aligned}
|u-v|_{+} & \leq \inf _{\epsilon \geq 0} \Phi_{+}(v ; \epsilon) \leq \Phi_{+}(v ; 0) \\
& =\sup _{(y, p) \in \mathcal{A}_{+}(v ; 0)}(R(v ; y, p))^{+}=\sup _{y \in[0,1)}\left(R\left(v ; y, v^{\prime}(y)\right)\right)^{+} \\
& =c^{2}(1-c) / 4(1+c)<(1-c) .
\end{aligned}
$$

This implies that ei $(u, v)=1$.

b. Nonsmooth $u$, nonoscillatory $R(v ; \cdot, \cdot)$. The case in which a sharp error estimate is most difficult to obtain is the case of a strictly nonlinear Hamiltonian and a viscosity solution with a kink. As an example, consider the Hamilton-Jacobi equation (1.1) with $d=1, H(p)=\frac{1}{2} p^{2}$, and $f(x)=\frac{1}{2}-|x|$; its viscosity solution is $u(x)=-|x|$. We take the function $v=v_{\nu}$ to be

$$
v_{\nu}(x)=-\nu \ln (\exp (x / \nu)+2+\exp (-x / \nu)),
$$

which is the solution of the parabolic equation

$$
v+\frac{1}{2}\left(v^{\prime}\right)^{2}-\nu v^{\prime \prime}=f_{\nu}
$$

where $f_{\nu}(x)=v_{\nu}(x)+1 / 2$.

In this case

$$
\left\|u-v_{\nu}\right\|_{\mathrm{L}^{\infty}}=\nu \ln 4
$$

that is, $v_{\nu}$ converges uniformly to $u$ and the convergence is of order one in the viscosity coefficient $\nu$.

Now, we apply our a posteriori error estimate. Of course this example is not periodic and we cannot apply Theorem 2.2 directly. However, in this particular case one can show directly that $\psi$ achieves a maximum-whereas periodicity is used to assert this in the proof of Theorem 2.2-and from there the proof can proceed as given.

All the computations below can be rigorously justified for $\nu \leq 1 / 2$. We begin by estimating $\left|u-v_{\nu}\right|_{-}$. By Theorem 2.2 , we have

$$
\left|u-v_{\nu}\right|_{-} \leq \inf _{\epsilon \geq 0} \Phi_{-}\left(v_{\nu} ; \epsilon\right)=\inf _{\epsilon \geq 0} \sup _{(y, p) \in \mathcal{A}_{-}\left(v_{\nu} ; \epsilon\right)}\left(-R_{-\epsilon}\left(v_{\nu} ; y, p\right)\right)^{+} .
$$

Since

$$
\mathcal{A}_{-}\left(v_{\nu} ; \epsilon\right)=\left\{\left(y, v_{\nu}^{\prime}(y)\right):|y| \geq y_{\epsilon, \nu}\right\}
$$

where $y_{\epsilon, \nu} \geq 0$ is the biggest root of the equation $z=-\epsilon v_{\nu}^{\prime}(z)$ (see Figure 2), we get

$$
\left|u-v_{\nu}\right|_{-} \leq \inf _{\epsilon \geq 0} \sup _{y \geq y_{\epsilon, \nu}}\left(-R_{-\epsilon}\left(v_{\nu} ; y, v_{\nu}^{\prime}(y)\right)\right)^{+}=\inf _{\epsilon \geq 0}\left(-R_{-\epsilon}\left(v_{\nu} ; y_{\epsilon, \nu}, v_{\nu}^{\prime}\left(y_{\epsilon, \nu}\right)\right)\right)^{+} .
$$




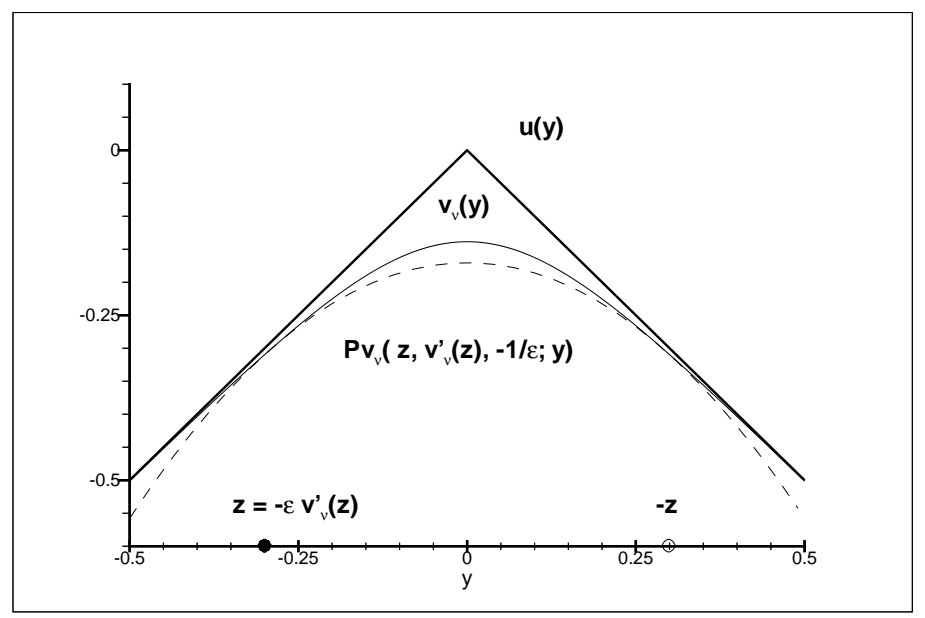

Figure 2. The viscosity solution $u$, its approximation $v_{\nu}$ and the parabola $y \mapsto P v_{\nu}\left(z, v_{\nu}^{\prime}(z),-1 / \epsilon ; y\right)$ for $z=y_{\epsilon, \nu}$ and $\nu=0.1$.

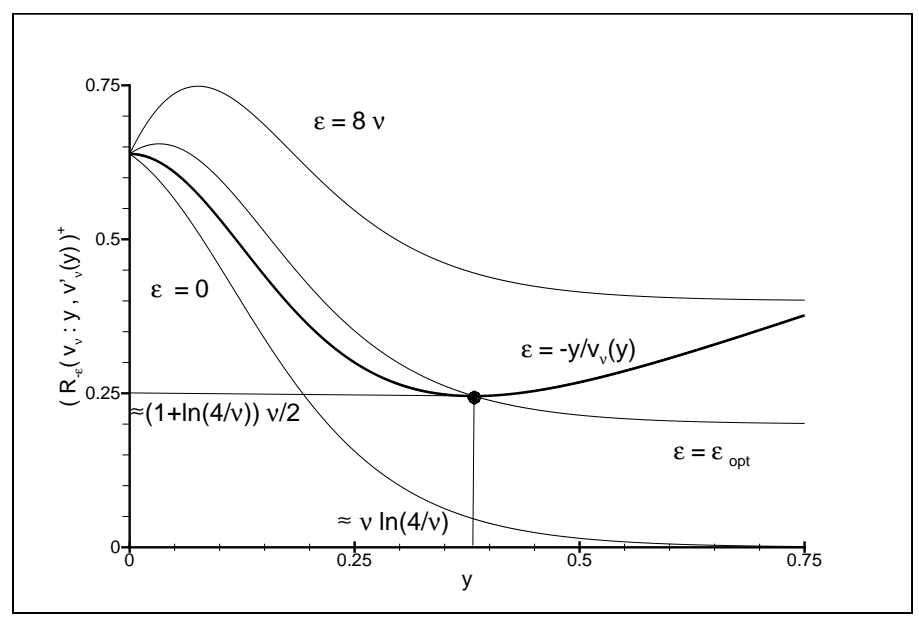

Figure 3 . The functions $y \mapsto\left(-R_{-\epsilon}\left(v_{\nu} ; y, v_{\nu}^{\prime}(y)\right)\right)^{+}$for three values of $\epsilon$ (thin lines) and the function $y \mapsto$ $\left(-R_{-y / v_{\nu}^{\prime}(y)}\left(v_{\nu} ; y, v_{\nu}^{\prime}(y)\right)\right)^{+}($thick line $)$for $\nu=0.1$.

In Figure 3, the functions $y \mapsto\left(-R_{-\epsilon}\left(v_{\nu} ; y, v_{\nu}^{\prime}(y)\right)\right)^{+}$are plotted for $y \geq 0$, for three values of $\epsilon$ and for $\nu=0.1$. Note how the shifted residual at $y,\left(-R_{-\epsilon}\left(v_{\nu} ; y, v_{\nu}^{\prime}(y)\right)\right)^{+}$, increases with $\epsilon$. The thick line represents the function

$$
y_{\epsilon, \nu} \mapsto\left(-R_{-\epsilon}\left(v_{\nu} ; y_{\epsilon, \nu}, v_{\nu}^{\prime}\left(y_{\epsilon, \nu}\right)\right)\right)^{+},
$$

which, by the definition of $y_{\epsilon, \nu}$, coincides with the function

$$
y \mapsto\left(-R_{-y / v_{\nu}^{\prime}(y)}\left(v_{\nu} ; y, v_{\nu}^{\prime}(y)\right)\right)^{+} .
$$

Clearly, this function intersects the functions $y \mapsto\left(-R_{-\epsilon}\left(v_{\nu} ; y, v_{\nu}^{\prime}(y)\right)\right)^{+}$at the abscissae $y=y_{\epsilon, \nu}$. Note how the value $y_{\epsilon, \nu}$ increases as $\epsilon$ increases; this reflects the fact that the size of $\mathcal{A}_{-}\left(v_{\nu} ; \epsilon\right)$ decreases as $\epsilon$ increases. 
It is a simple but long exercise to show that

$$
\epsilon_{\mathrm{opt}}(\nu)=\nu \log (4 / \nu)(1+\mathcal{O}(\nu))
$$

This gives that

$$
y_{\epsilon_{\mathrm{opt}}(\nu), \nu}=\nu \log (4 / \nu)(1+\mathcal{O}(\nu))
$$

and that

$$
\left|u-v_{\nu}\right|_{-} \leq \frac{\nu}{2}(1+\ln (4 / \nu))(1+\mathcal{O}(\nu)) .
$$

The evaluation of $\left|u-v_{\nu}\right|_{+}$is very simple and will not be presented; it gives $\left|u-v_{\nu}\right|_{+}=0$, as expected. This implies that

$$
\mathrm{ei}\left(u, v_{\nu}\right)=\frac{1+\ln (4 / \nu)}{\ln 16}(1+\mathcal{O}(\nu))
$$

Unfortunately, in this case the value of ei $\left(u, v_{\nu}\right)$ is bigger than the optimal value of one, but, on the other hand, it is a quantity that remains between 1 and 6 as the viscosity coefficient $\nu$ varies from $1 / 2$ to $10^{-6}$; this is extremely good for practical purposes.

We end these computations by seeing what would have happened if we had used $\Phi_{\sigma}\left(v_{\nu} ; 0\right)$ instead of $\Phi_{\sigma}\left(v_{\nu} ; \epsilon_{\mathrm{opt}}(\nu)\right)$. A simple computation gives

$$
\Phi_{\sigma}\left(v_{\nu} ; 0\right)= \begin{cases}-R\left(v_{\nu} ; 0,0\right)=1 / 2+\nu \ln (4) & \text { if } \sigma=-, \\ 0 & \text { if } \sigma=+,\end{cases}
$$

and so, we would have obtained the upper bound

$$
\max _{\sigma \in\{-1,+1,\}} \Phi_{\sigma}\left(v_{\nu} ; 0\right)=1 / 2+\nu \ln (4),
$$

which is totally useless from the practical point of view! The fact that the residual at $y=0$ does not go to zero as the diffusion coefficient $\nu$ goes to zero is a reflection of the presence of a kink in the viscosity solution. In other words, it is impossible to drive the residual to zero pointwise in the presence of kinks in the viscosity solution. This is why it is essential to look for the optimal value of the auxiliary parameter $\epsilon$.

c. A very special case and a highly-oscillatory $R(v ; \cdot, \cdot)$. Consider the very special case when $f \equiv c$; the viscosity solution of equation (1.1) is thus $u \equiv c-H(0)$. Let $v_{N}$ be any continuous and periodic function with period 1 .

We begin by estimating $\left|u-v_{N}\right|_{-}$. By Theorem 2.2, we have

$$
\left|u-v_{N}\right|_{-} \leq \inf _{\epsilon \geq 0} \Phi_{-}\left(v_{N} ; \epsilon\right)=\inf _{\epsilon \geq 0} \sup _{(y, p) \in \mathcal{A}_{-}\left(v_{N} ; \epsilon\right)}\left(-R_{-\epsilon}\left(v_{N} ; y, p\right)\right)^{+} .
$$

Since $\epsilon \geq \epsilon^{\prime}$ implies that

$$
\begin{aligned}
\mathcal{A}_{-}\left(v_{N} ; \epsilon\right) & \subset \mathcal{A}_{-}\left(v_{N} ; \epsilon^{\prime}\right), \\
\left(-R_{-\epsilon}\left(v_{N} ; y, p\right)\right)^{+} & \leq\left(-R_{-\epsilon^{\prime}}\left(v_{N} ; y, p\right)\right)^{+},
\end{aligned}
$$

we have

$$
\Phi_{-}\left(v_{N} ; \epsilon\right) \leq \Phi_{-}\left(v_{N} ; \epsilon^{\prime}\right) \quad \text { if } \epsilon \geq \epsilon^{\prime}
$$

and hence

$$
\left|u-v_{N}\right|_{-} \leq \lim _{\epsilon \rightarrow \infty} \Phi_{-}\left(v_{N} ; \epsilon\right)
$$


Finally, since

$$
A_{-}\left(v_{N} ; \infty\right)=\left\{(x, 0): v_{N}(x) \leq v_{N}(y) \forall y \in \mathbb{R}\right\} \equiv \mathcal{X} \times\{0\},
$$

we get

$$
\begin{aligned}
\left|u-v_{N}\right|_{-} & \leq \sup _{x \in \mathcal{X}}\left(-v_{N}(x)-H(0)+f\right)^{+} \\
& =\sup _{x \in \mathcal{X}}\left(u-v_{N}(x)\right)^{+} \\
& =\sup _{x \in \mathbb{R}}\left(u-v_{N}(x)\right)^{+} \\
& =\left|u-v_{N}\right|_{-} .
\end{aligned}
$$

A similar result holds for $\left|u-v_{N}\right|_{+}$, and so we get the optimal result ei $\left(u, v_{N}\right)=1$.

Let us see what would had happened if we had used $\Phi_{\sigma}\left(v_{N} ; 0\right)$ instead of $\Phi_{\sigma}\left(v_{N} ; \infty\right)$ to estimate the error; we take $v_{N}$ to be the following highly oscillatory approximation:

$$
v_{N}(y)=c-\frac{1}{N^{2}} \cos (2 \pi N y)
$$

Since

$$
R\left(v_{N} ; y, v_{N}^{\prime}(y)\right)=-\frac{1}{N^{2}} \cos (2 \pi N y)+H\left(\frac{2 \pi}{N} \sin (2 \pi N y)\right),
$$

we have, for general Lipschitz Hamiltonians $H$,

$$
\max _{\sigma \in\{-1,+1,\}} \Phi_{\sigma}\left(v_{N} ; 0\right)=\mathcal{O}(1 / N),
$$

and so we would have obtained an $\mathcal{O}(N)$ times bigger upper bound!

d. An analogy. It is well known that extra care is needed when obtaining error estimates for an approximate solution with a highly oscillatory residual. Next, we briefly illustrate this point. If $u$ is the weak solution of the following boundary value problem:

$$
-\Delta u=f \quad \text { in } \Omega, \quad u=0 \quad \text { on } \partial \Omega,
$$

the following error estimate can be proved:

$$
\|u-v\|_{H_{0}^{1}(\Omega)} \leq C\|R(v)\|_{H^{-1}(\Omega)},
$$

where $v$ is any function in $H_{0}^{1}(\Omega), R(v)=-\Delta v-f$, and $C$ is a constant that depends on $\Omega$ only. Since the norm in $H^{-1}(\Omega)$ is difficult to compute, we can use the crude estimate

$$
\|R(v)\|_{H^{-1}(\Omega)} \leq\|R(v)\|_{L^{2}(\Omega)}
$$

to get the new upper bound

$$
\|u-v\|_{H_{0}^{1}(\Omega)} \leq C\|R(v)\|_{L^{2}(\Omega)},
$$

which now is in terms of an easy-to-compute norm of the residual of $v, R(v)$. This naïve approach, however, does not take into account the possible oscillatory behavior of the residual $R(v)$. On the other hand, if $v$ is, for example, a $\mathcal{C}^{1}$ function determined by a Galerkin method in a mesh of squares of size $1 / N$, it can be shown that

$$
\|R(v)\|_{H^{-1}(\Omega)} \leq \mathcal{C} N^{-1}\|R(v)\|_{L^{2}(\Omega)},
$$


where $\mathcal{C}$ is a computable constant independent of $N$. This finer estimate does take into account the oscillatory behavior of the residual $R(v)$, typical of Galerkin methods, and results in the much better estimate

$$
\|u-v\|_{H_{0}^{1}(\Omega)} \leq C \mathcal{C} N^{-1}\|R(v)\|_{L^{2}(\Omega)} .
$$

In our framework, the question is if the possible oscillatory behavior of the residual $R(v ; \cdot, \cdot)$ can be captured by means of the search for the optimal parameter $\epsilon$, the use of the shifted residual $R_{\sigma \epsilon}(v ; \cdot, \cdot)$, and the definition of the set $\mathcal{A}_{\sigma}(v ; \epsilon)$. The previous example shows that in some cases (smooth oscillatory residual), this is the case. Unfortunately, this behavior of our a posteriori error estimate does not seem to hold in general. A way around this difficulty is to apply the estimate to a post-processed approximate solution. The purpose of the post-processing is to eliminate the oscillations in the residual while maintaining the quality of the approximation. In the next section, we show that this strategy works very well.

\section{Numerical EXPERIMENTS}

The purpose of this section is to study the application of our a posteriori error estimator to approximations generated through numerical schemes; we consider a simple, standard monotone scheme and the modern high-resolution DG method of $\mathrm{Hu}$ and Shu 10 . We evaluate the effectivity indexes for these schemes on several prototypical one-dimensional problems. Finally, to show that results similar to the ones obtained in this case can also be obtained in two dimensions, we include a couple of experiments with monotone schemes in two space dimensions. For the sake of simplicity, we use uniform grids in all our experiments.

4.1. Discretization of the norms and the nonlinear functionals of the a posteriori error estimate. Since the approximate solutions are defined by a finite number of degrees of freedom, it is reasonable, in practice, to replace the domain $\Omega=[0,1)^{d}$ over which we evaluate the seminorms $|\cdot|_{\sigma}$ and the functionals $\Phi_{\sigma}(\cdot, \cdot)$, by a finite number of points in $\Omega$ which we denote by $\Omega_{h}$.

Another modification to be made in practice concerns the values of the auxiliary parameter $\epsilon$. Theoretically, the evaluation of $\Phi_{\sigma}(\cdot, \cdot)$ requires optimization over the set $\epsilon \in[0, \infty)$. In practice, however, it is sensible to replace $[0, \infty)$ by the following set:

$$
\mathcal{E}_{h}=\{i \cdot E / N, 0 \leq i \leq N\},
$$

where

$$
E=2 \omega|\ln (1 / \omega)|, \quad N=4|\ln (1 / \omega)|,
$$

where $\omega$ is an upper bound for the artificial diffusion coefficient of the numerical scheme under consideration. The above choice of $E$ is motivated by the second analytic example of Section 3. Indeed, in this example, in which the viscosity solution has a kink, the optimal value of $\epsilon$ is, approximately, $\nu|\ln (1 / \nu)|$; since $\nu$ is the diffusion coefficient, it is reasonable to take $E=\omega|\ln (1 / \omega)|$, where $\omega$ is the maximum artificial diffusion coefficient of the numerical scheme. To be on the safe side, we multiply this number by two. The choice of $N$ is somewhat arbitrary, but we have found it to work well in practice. 
We denote by $|\cdot|_{h, \sigma}$ and $\Phi_{h, \sigma}(\cdot)$ the corresponding quantities obtained after the above mentioned modifications. The effectivity index ei $(u, v)$ is, accordingly, replaced by what we could call the computational effectivity index

$$
\operatorname{ei}_{h}(u, v)=\max _{\sigma \in\{+,-\}} \Phi_{h, \sigma}(v) / \max _{\sigma \in\{+,-\}}|u-u|_{h, \sigma}
$$

The main objective of our numerical experiments is to study the performance of this index.

4.2. Fast evaluation of the paraboloid test. To evaluate the condition (2.8) is computationally very expensive, since to determine if the point $(x, p)$ belongs to the set $\mathcal{A}_{\sigma}(v ; \epsilon)$ we must compare $v(y)$ and $P_{v}(x, p, \sigma / \epsilon ; y)$ for each point $y$ in $\Omega$. Fortunately, when $v$ is Lipschitz in $\Omega$, which is the case in most practical applications, it is not necessary to perform this comparison for $y$ in the whole domain $\Omega$ but only on a significantly smaller set. To see this, note that if the paraboloid $P_{v}(x, p, \sigma / \epsilon ; \cdot)$ is 'tangent' to $v$ at the point $y$, we must have that

$$
q=p+\sigma(y-x) / \epsilon,
$$

for some $q \in D^{\sigma} v(y)$. This implies that

$$
|y-x| \leq|p-q| \epsilon \leq 2\|v\|_{\operatorname{Lip}(\Omega)} \epsilon .
$$

This means that we can replace the condition (2.8) by the following:

$$
\sigma\left\{v(y)-P_{v}(x, p, \sigma / \epsilon ; y)\right\} \leq 0 \quad \forall y \in \Omega:|y-x| \leq 2\|v\|_{\operatorname{Lip}(\Omega)} \epsilon .
$$

4.3. The discrete paraboloid test. In our computations, we actually use the following discrete version of the above paraboloid test:

$$
\sigma\left\{v(y)-P_{v}(x, p, \sigma / \epsilon ; y)\right\} \leq 0 \quad \forall y \in \Omega_{h}:|y-x| \leq 2\|v\|_{\operatorname{Lip}(\Omega)} \epsilon,
$$

which is carried out only for $x \in \Omega_{h}$, of course. Note that by using this version of the paraboloid test, the computationl cost of evaluating the discrete a posteriori error estimator for a value of $\epsilon \in \mathcal{E}_{h}$ at a given point of $\Omega_{h}$ is proportional to

$$
\left(\|v\|_{\operatorname{Lip}(\Omega)} \frac{\omega}{\Delta x}|\ln \omega|\right)^{d},
$$

if we assume that $\Omega_{h}$ is a uniform Cartesian grid of $d$-dimensional cubes of side $\Delta x$. Note that this evaluation can be done in parallel; if this is the case, since there are only $4|\ln \omega|+1$ points in $\mathcal{E}_{h}$ the whole computation can be carried out in a number of operations proportional to

$$
\left(\|v\|_{\operatorname{Lip}(\Omega)} \frac{\omega}{\Delta x}|\ln \omega|\right)^{d}|\ln \omega| .
$$

Since $\omega$ is proportional to $\Delta x$, the computational complexity of evaluating the discrete a posteriori error estimate is only proportional to

$$
\|v\|_{\operatorname{Lip}(\Omega)}^{d}|\ln \Delta x|^{d+1} .
$$


TABLE 1. Smooth solution test problems.

\begin{tabular}{|c|c|c|}
\hline Hamiltonian $H(p)$ & right-hand side $f(x)$ & viscosity solution $u(x)$ \\
\hline $\begin{array}{ll}p & \text { (linear) }\end{array}$ & $\cos ^{2}(\pi x)-\pi \sin (2 \pi x)$ & $\cos ^{2}(\pi x)$ \\
\hline$-p^{2} / 4 \pi^{2} \quad$ (concave) & $\cos ^{4}(\pi x)$ & $\cos ^{2}(\pi x)$ \\
\hline$p^{3} / 8 \pi^{3} \quad$ (nonconvex) & $\sin (2 \pi x)+\cos ^{3}(2 \pi x)$ & $\sin (2 \pi x)$ \\
\hline
\end{tabular}

TABLE 2. Nonsmooth solution test problems.

\begin{tabular}{ccc}
\hline Hamiltonian $H(p)$ & right-hand side $f(x)$ & viscosity solution $u(x)$ \\
\hline $\begin{array}{c}p^{2} / \pi^{2} \\
(\text { convex })\end{array}$ & $-|\cos (\pi x)|+\sin ^{2}(\pi x)$ & $-|\cos (\pi x)|$ \\
$\begin{array}{c}p^{4}+2 p^{2}-1 \\
\text { (nonconvex) }\end{array}$ & $u(x)+H\left(u^{\prime}(x)\right)$ if $x \neq 1 / 2$ & $\left\{\begin{array}{l}x^{2}, \text { if } 0 \leq x \leq \frac{1}{2}, \\
(x-1)^{2}, \text { if } \frac{1}{2} \leq x \leq 1 .\end{array}\right.$ \\
\hline
\end{tabular}

\subsection{One-dimensional experiments.}

a. The test problems. We test our error estimator with the one-dimensional test problems described in Tables 1 and 2 . The solutions of the problems displayed in Table 1 are smooth, and the solutions of the problems in Table 2 both have kinks at $x=1 / 2$.

b. Monotone schemes. We use the following monotone scheme defined on a uniform grid:

$$
\begin{aligned}
& v_{j}+H\left(\frac{v_{j+1}-v_{j-1}}{2 \Delta x}\right)-\omega \frac{v_{j+1}-2 v_{j}+v_{j-1}}{\Delta x^{2}}=f_{j}, \\
& \omega=\sup _{x \in \Omega} \frac{\Delta x}{2}\left|H^{\prime}\left(f^{\prime}(x)\right)\right|,
\end{aligned}
$$

where $\phi_{j} \equiv \phi\left(x_{j}\right)$ and $x_{j}=j \Delta x$. The term $\omega$ allows the artificial viscosity to scale with the discretization. The upside to the use of monotone schemes is that we are guaranteed their convergence to the correct viscosity solution, cf. Crandall and Lions [7. The downside is that they are at most first order accurate.

Our a posteriori error estimator is based on the notion of viscosity solutions and so requires comparison between continuous functions. A natural choice for $v$ is the classical piecewise linear interpolant of the numerical solution at the points $x_{j}$. Choosing $\Omega_{h}=\left\{x_{j+1 / 2}\right\}$ then leads to a well-defined derivative at the points where the solution is sampled. Thus, the shifted residual $R_{\sigma \epsilon}\left(v ; x_{j+1 / 2}, v^{\prime}\left(x_{j+1 / 2}\right)\right)$ becomes

$$
v\left(x_{j+1 / 2}\right)+H\left(v^{\prime}\left(x_{j+1 / 2}\right)\right)-f\left(x_{j+1 / 2}-\sigma \epsilon v^{\prime}\left(x_{j+1 / 2}\right)\right)-\frac{1}{2} \sigma \epsilon\left|v^{\prime}\left(x_{j+1 / 2}\right)\right|^{2},
$$

where $v^{\prime}\left(x_{j+1 / 2}\right)=\frac{v_{j+1}-v_{j}}{\Delta x}$.

Next, we describe our numerical results. In Table 3, we show our results for the smooth solution test problems. We see that in each of the three problems, the monotone scheme converges linearly, as expected, and that the computational effectivity index is independent of the mesh step size $\Delta x$; moreover, we see that for 
TABLE 3. Monotone scheme on smooth solution test problems.

\begin{tabular}{ccccc}
\hline Hamiltonian & $1 / \Delta x$ & error & order & $\mathrm{ei}_{h}(u, v)$ \\
\hline linear & 40 & $3.8 e-2$ & - & 6.4 \\
& 80 & $1.9 e-2$ & 0.99 & 6.4 \\
& 160 & $9.7 e-3$ & 1.00 & 6.4 \\
& 320 & $4.8 e-3$ & 1.00 & 6.4 \\
& 640 & $2.4 e-3$ & 1.00 & 6.4 \\
& 1280 & $1.2 e-3$ & 1.00 & 6.4 \\
\hline concave & 40 & $1.4 e-1$ & - & 1.0 \\
& 80 & $7.5 e-2$ & 0.91 & 1.0 \\
& 160 & $3.8 e-2$ & 0.96 & 1.0 \\
& 320 & $1.9 e-2$ & 0.98 & 1.0 \\
& 640 & $9.8 e-3$ & 0.99 & 1.0 \\
& 1280 & $4.9 e-3$ & 1.00 & 1.0 \\
\hline nonconvex & 40 & $5.0 e-1$ & - & 1.3 \\
& 80 & $3.1 e-1$ & 0.69 & 1.4 \\
& 160 & $1.8 e-1$ & 0.81 & 1.4 \\
& 320 & $9.7 e-2$ & 0.86 & 1.3 \\
& 640 & $5.2 e-2$ & 0.90 & 1.2 \\
& 1280 & $2.7 e-2$ & 0.93 & 1.1 \\
\hline
\end{tabular}

TABLE 4. Monotone scheme on nonsmooth solution test problems.

\begin{tabular}{ccccc}
\hline Hamiltonian & $1 / \Delta x$ & error & order & $\mathrm{ei}_{h}(u, v)$ \\
\hline convex & 40 & $1.5 e-1$ & - & 1.8 \\
& 80 & $7.7 e-2$ & 0.98 & 1.7 \\
& 160 & $3.9 e-2$ & 0.99 & 2.0 \\
& 320 & $2.0 e-2$ & 1.00 & 2.3 \\
& 640 & $9.8 e-3$ & 1.00 & 2.4 \\
& 1280 & $4.9 e-3$ & 1.00 & 2.9 \\
\hline nonconvex & 40 & $3.9 e-2$ & - & 1.5 \\
& 80 & $1.9 e-2$ & 1.00 & 1.8 \\
& 160 & $9.6 e-3$ & 1.00 & 2.6 \\
& 320 & $4.8 e-3$ & 1.00 & 3.5 \\
& 640 & $2.4 e-3$ & 1.00 & 4.6 \\
& 1280 & $1.2 e-3$ & 1.00 & 6.3 \\
\hline & & & &
\end{tabular}

nonlinear Hamiltonians, the computational effectivity indexes are nearly optimal. In all these case, the optimal value of $\epsilon$ was 0 , in agreement with our first analytic example.

The results for the nonsmooth solution test problems are displayed in Table 4 We can see that the scheme converges linearly, as expected, and the computational effectivity index remains reasonably small throughout the huge variation of the size of $\Delta x$. In the convex Hamiltonian case, we expected a behavior of the computational effectivity index similar to the one observed in the second analytic example. The fact that the computational effectivity index increases more slowly must be an effect of the discretization of the functionals $\Phi_{\sigma}(v, \epsilon)$. In the case of the nonconvex Hamiltonian, we see, however, a more marked though slow increase in the computational effectivity index, as we would expect. 
TABlE 5. Monotone scheme on nonsmooth solution test problems: The set $[a, b]$ for which the parabola test failed, the number of grid points in the interval, and the optimal value $\epsilon_{\mathrm{opt}}$.

\begin{tabular}{cccccccc}
\hline Hamiltonian & $1 / \Delta x$ & $a$ & $b$ & $|b-a| / \Delta x$ & $\epsilon_{\mathrm{opt}}$ & $\epsilon_{\mathrm{opt}} / E$ & $N \cdot \epsilon_{\mathrm{opt}} / E$ \\
\hline convex & 40 & .425 & .550 & 5 & $3.1 e-2$ & 0.24 & 4 \\
& 80 & .450 & .538 & 7 & $1.6 e-2$ & 0.21 & 4 \\
& 160 & .475 & .519 & 7 & $8.0 e-3$ & 0.18 & 4 \\
& 320 & .484 & .513 & 9 & $5.0 e-3$ & 0.20 & 5 \\
& 640 & .492 & .506 & 9 & $2.5 e-3$ & 0.18 & 5 \\
& 1280 & .495 & .504 & 11 & $1.5 e-3$ & 0.20 & 6 \\
\hline nonconvex & 40 & .475 & .500 & 1 & $2.9 e-2$ & 0.20 & 3 \\
& 80 & .475 & .513 & 3 & $2.4 e-2$ & 0.27 & 5 \\
& 160 & .488 & .506 & 3 & $1.2 e-2$ & 0.23 & 5 \\
& 320 & .491 & .506 & 5 & $9.6 e-3$ & 0.33 & 8 \\
& 640 & .494 & .505 & 7 & $6.0 e-3$ & 0.37 & 10 \\
& 1280 & .496 & .503 & 9 & $3.9 e-3$ & 0.44 & 13 \\
\hline
\end{tabular}

In Table 5, we show the numbers $\epsilon_{\mathrm{opt}} / E$ and $N \cdot \epsilon_{\mathrm{opt}} / E$ which indicate what fraction of $E$ is the optimal auxiliary parameter $\epsilon_{\mathrm{opt}}$ and on how many values of $\epsilon$ the nonlinear functional of the error estimate have to be evaluated before hitting their minimum, respectively. It can be seen that our choices of the parameters $E$ and $N$ are quite reasonable, since they change very little as the discretization parameter $\Delta x$ changes several orders of magnitude. In Table 5 , we also display the interval over which the parabola test failed, that is, the interval containing the set of points of $\Omega_{h}$ for which the condition (4.1) is not satisfied. Note how this set always contains the point $x=1 / 2$ at which the viscosity solution has its only kink, and shrinks as the mesh size $\Delta x$ goes to zero. This indicates that the parabola test can be used to give an indication of the location of the kinks of the exact solution.

c. Discontinuous Galerkin schemes. We adapt the DG method of $\mathrm{Hu}$ and Shu [10], originally devised for transient Hamilton-Jacobi problems, to our steady state setting. Next, we briefly describe this DG method; see also the short monograph on DG methods [3] and the references therein. We start by seeking a steady state solution to the equation

$$
u_{t}+u+H\left(u_{x}\right)-f=0 .
$$

After differentiating the above with respect to $x$, we obtain the equation

$$
\phi_{t}+\phi+H(\phi)_{x}-f_{x}=0 .
$$

for $\phi=u_{x}$. This may be viewed as a conservation law with both source and forcing terms included. As such, we apply the DG method. Allowing our initial condition to evolve to a steady state gives us the solution $\phi$ to

$$
\phi+H(\phi)_{x}-f_{x}=0
$$

which is then integrated to recover the solution to our original steady state Hamilton-Jacobi equation. 
The solution to (4.4) is based on a weak formulation. Specifically, we multiply $\phi$ by a test function and then integrate by parts on the spatial derivative, obtaining on each interval

$$
\int_{x_{j}}^{x_{j+1}}\left(\phi_{t} v+\phi v-H(\phi) v_{x}-f_{x} v\right) d x+H\left(\phi\left(x_{j+1}^{-}\right)\right) v\left(x_{j+1}^{-}\right)-H\left(\phi\left(x_{j}^{+}\right)\right) v\left(x_{j}^{+}\right)=0 .
$$

The flux terms $H(p)$ appearing outside the integral are then replaced by an appropriate choice of numerical flux, $\widehat{H}\left(\phi\left(x_{j}^{-}\right), \phi\left(x_{j}^{+}\right)\right)$. We take the Lax-Friedrichs flux, namely,

$$
\widehat{H}^{L F}(a, b)=\frac{1}{2}[H(a)+H(b)-C(b-a)], \quad C=\max _{\inf \phi(x, 0) \leq s \leq \sup \phi(x, 0)}\left|H^{\prime}(s)\right| .
$$

The above weak formulation is used to define an approximate solution $\phi_{h}$ which at each time is a piecewise polynomial of degree $k$. Then a Runge-Kutta time discretization is used to drive the solution to the steady state; note that for degree zero polynomial approximation $(k=0)$ this reduces to the well-known Lax-Friedrichs scheme.

Next, we describe how we adapt this DG method to steady state problems. Since the viscosity solution to a Hamilton-Jacobi equation may have a discontinuity in its derivative, one must deal with the problems caused by shocks in the solution of the corresponding conservation law. One typically implements slope limiters by using a test to determine whether there is too much oscillation being generated in a given region of the computational domain. The emerging spurious oscillation is then eliminated by suitably lowering the degree of the approximating polynomial. This region may change throughout the course of a computation, and even if at one timestep a given element is using a low order approximation, this same element is allowed to use a higher order approximation later on. However, it is very well known that this freedom does hurt the convergence to the steady state, since the iterative sequence typically falls into a limit cycle. To deal with this difficulty we first use a standard slope limiting method up to a point where the residual ceases to decrease; thereafter we continue the time stepping but do not allow the degree of polynomial approximation on a given element to increase once it had been reduced.

Next, we discuss our results. In all our experiments, we keep the choice of $E$ and $N$ we took for the monotone scheme. For piecewise-constant approximations, $k=0$, we take $\Omega_{h}=\left\{x_{j+1 / 2}\right\}$. It is well known that in this case, the DG method reduces to a monotone scheme; its solution approximates a parabolic regularization of the conservation law which has nonoscillatory residual. As a consequence, we expect the numerical results to be similar to those obtained with the monotone scheme. A glance at Tables [6, 7 and 8 shows that this is indeed the case.

In the case of piecewise polynomial approximation of degree $k>0$, as is typical of finite element approximations, the residual becomes increasingly oscillatory as $k$ increases. For these kinds of residuals, the effectivity index can grow like $(\Delta x)^{-1}$, as was illustrated in our third analytic example. This is precisely what we observe in Table 9] where results for $k=1$ and smooth solutions are shown. Here we sampled at $\Omega_{h}=\left\{x_{j+1 / 4}, x_{j+3 / 4}\right\}$. To remedy this situation, we post-process the approximate solution in an effort to filter out the oscillations in the residual while keeping the order of accuracy of the approximation invariant. We use the 
TABlE 6 . DG method with $k=0$ on smooth solution test problems.

\begin{tabular}{ccccc}
\hline Hamiltonian & $1 / \Delta x$ & error & order & $\operatorname{ei}_{h}(u, v)$ \\
\hline linear & 40 & $3.9 e-2$ & - & 6.4 \\
& 80 & $1.9 e-2$ & 0.99 & 6.4 \\
& 160 & $9.7 e-3$ & 1.00 & 6.4 \\
& 320 & $4.8 e-3$ & 1.00 & 6.4 \\
& 640 & $2.4 e-3$ & 1.00 & 6.4 \\
& 1280 & $1.2 e-3$ & 1.00 & 6.4 \\
\hline concave & 40 & $1.4 e-1$ & - & 1.0 \\
& 80 & $7.4 e-2$ & 0.91 & 1.0 \\
& 160 & $3.8 e-2$ & 0.96 & 1.0 \\
& 320 & $1.9 e-2$ & 0.98 & 1.0 \\
& 640 & $9.8 e-3$ & 0.99 & 1.0 \\
& 1280 & $4.9 e-3$ & 1.00 & 1.0 \\
\hline nonconvex & 40 & $5.0 e-1$ & - & 1.3 \\
& 80 & $3.1 e-1$ & 0.69 & 1.4 \\
& 160 & $1.8 e-1$ & 0.81 & 1.4 \\
& 320 & $9.7 e-2$ & 0.86 & 1.3 \\
& 640 & $5.2 e-2$ & 0.90 & 1.2 \\
& 1280 & $2.7 e-2$ & 0.93 & 1.1 \\
\hline
\end{tabular}

TABLE 7. DG method with $k=0$ on nonsmooth solution test problems.

\begin{tabular}{ccccc}
\hline Hamiltonian & $1 / \Delta x$ & error & order & $\mathrm{ei}_{h}(u, v)$ \\
\hline convex & 40 & $1.5 e-1$ & - & 1.9 \\
& 80 & $7.7 e-2$ & 0.97 & 1.7 \\
& 160 & $3.9 e-2$ & 0.99 & 2.1 \\
& 320 & $2.0 e-2$ & 0.99 & 2.3 \\
& 640 & $9.8 e-3$ & 1.00 & 2.6 \\
& 1280 & $4.9 e-3$ & 1.00 & 2.9 \\
\hline nonconvex & 40 & $3.8 e-2$ & - & 1.5 \\
& 80 & $1.9 e-2$ & 0.98 & 1.8 \\
& 160 & $9.6 e-3$ & 0.99 & 2.3 \\
& 320 & $4.8 e-3$ & 1.00 & 3.5 \\
& 640 & $2.4 e-3$ & 1.00 & 4.6 \\
& 1280 & $1.2 e-3$ & 1.00 & 6.3 \\
\hline & & & &
\end{tabular}

convolution kernel $\frac{1}{\Delta x} K^{4,2}(x / \Delta x)$ defined by

$$
K^{4,2}(y)=-\frac{1}{12} \psi^{(2)}(y-1)+\frac{7}{6} \psi^{(2)}(y)-\frac{1}{12} \psi^{(2)}(y+1),
$$

where $\psi^{(2)}$ is the B-spline obtained by convolving the characteristic function of $[-1 / 2,1 / 2]$ with itself once; see the monograph by Wahlbin [18] and the references therein. That this strategy works can be seen in Table 10, where we also see that the computational effectivity indexes remain reasonably small in all three cases. Note that the DG method converges with order three with or without post-processing.

When the viscosity solution has a kink, there is no essential difference between the effectivity indexes of the original and the post-processed solutions as can be 
TABLE 8. DG method with $k=0$ on nonsmooth solution test problems: The set $[a, b]$ for which the parabola test failed and the optimal value $\epsilon_{\mathrm{opt}}$.

\begin{tabular}{cccccccc}
\hline Hamiltonian & $1 / \Delta x$ & $a$ & $b$ & $|b-a| / \Delta x$ & $\epsilon_{\mathrm{opt}}$ & $\epsilon_{\mathrm{opt}} / E$ & $N \cdot \epsilon_{\mathrm{opt}} / E$ \\
\hline convex & 40 & .438 & .563 & 5 & $3.2 e-2$ & 0.24 & 4 \\
& 80 & .456 & .544 & 7 & $1.6 e-2$ & 0.21 & 4 \\
& 160 & .478 & .522 & 7 & $8.0 e-3$ & 0.18 & 4 \\
& 320 & .486 & .514 & 9 & $5.0 e-3$ & 0.20 & 5 \\
& 640 & .493 & .507 & 9 & $2.5 e-3$ & 0.18 & 5 \\
& 1280 & .496 & .504 & 11 & $1.5 e-3$ & 0.20 & 6 \\
\hline nonconvex & 40 & .488 & .513 & 1 & $3.8 e-2$ & 0.25 & 4 \\
& 80 & .481 & .519 & 3 & $2.4 e-2$ & 0.27 & 5 \\
& 160 & .491 & .509 & 3 & $1.2 e-2$ & 0.23 & 5 \\
& 320 & .492 & .508 & 5 & $9.6 e-3$ & 0.33 & 8 \\
& 640 & .495 & .506 & 7 & $6.0 e-3$ & 0.37 & 10 \\
& 1280 & .497 & .504 & 9 & $3.9 e-3$ & 0.44 & 13 \\
\hline
\end{tabular}

TABLE 9. DG method with $k=1$ on smooth solution test problems.

\begin{tabular}{ccccc}
\hline Hamiltonian & $1 / \Delta x$ & error & order & $\mathrm{ei}_{h}(u, v)$ \\
\hline linear & 40 & $4.5 e-5$ & - & 89 \\
& 80 & $5.6 e-6$ & 3.00 & 179 \\
& 160 & $7.0 e-7$ & 3.00 & 359 \\
& 320 & $8.8 e-8$ & 3.00 & 717 \\
& 640 & $1.1 e-8$ & 2.99 & 1423 \\
& 1280 & $2.7 e-9$ & 2.04 & 1466 \\
\hline concave & 40 & $2.0 e-5$ & - & 13 \\
& 80 & $2.6 e-6$ & 3.00 & 26 \\
& 160 & $3.2 e-7$ & 3.00 & 51 \\
& 320 & $4.0 e-8$ & 3.00 & 100 \\
& 640 & $5.0 e-9$ & 3.00 & 200 \\
& 1280 & $4.8 e-10$ & 3.37 & 518 \\
\hline nonconvex & 40 & $4.5 e-5$ & - & 34 \\
& 80 & $5.9 e-6$ & 2.94 & 66 \\
& 160 & $7.5 e-7$ & 2.97 & 129 \\
& 320 & $9.5 e-8$ & 2.98 & 256 \\
& 640 & $1.2 e-8$ & 2.99 & 509 \\
& 1280 & $1.5 e-9$ & 2.99 & 1015 \\
\hline
\end{tabular}

seen by comparing Tables 11 and 12 . This is because the error concentrates around the kink. Around it, the DG uses a low-degree polynomial approximation which gives rise to a locally nonoscillatory residual. As a consequence, we can expect results similar to the ones obtained with $k=0$ in these test problems. That this is indeed the case can be seen in Tables 12 and 13, where we report the results for the post-processed DG approximations. Note that the computational effectivity indexes vary very slowly as the discretization parameter varies a couple of orders of magnitude.

Comparing the results for the $k=0$ approximation in Tables $[7$ and 8 with the ones for the $k=1$ approximation in Tables 12 and 13 we note that (i) the $k=1$ 
TABLE 10. Post-processed DG method with $k=1$ on smooth solution test problems.

\begin{tabular}{ccccc}
\hline Hamiltonian & $1 / \Delta x$ & error & order & $\mathrm{ei}_{h}(u, v)$ \\
\hline linear & 40 & $2.7 e-5$ & - & 6.4 \\
& 80 & $3.3 e-6$ & 3.00 & 6.4 \\
& 160 & $4.2 e-7$ & 3.00 & 6.4 \\
& 320 & $5.2 e-8$ & 2.99 & 6.3 \\
& 640 & $6.6 e-9$ & 2.98 & 6.3 \\
& 1280 & $2.1 e-9$ & 1.63 & 3.5 \\
\hline concave & 40 & $4.5 e-6$ & - & 2.2 \\
& 80 & $5.8 e-7$ & 2.95 & 1.9 \\
& 160 & $7.5 e-8$ & 2.97 & 1.7 \\
& 320 & $9.4 e-9$ & 2.98 & 1.7 \\
& 640 & $1.2 e-9$ & 2.99 & 1.6 \\
nonconvex & 1280 & $3.5 e-12$ & 8.41 & 2.3 \\
\hline & 40 & $1.8 e-5$ & - & 4.1 \\
& 80 & $1.9 e-6$ & 3.25 & 4.3 \\
& 160 & $2.5 e-7$ & 2.91 & 3.7 \\
& 320 & $3.2 e-8$ & 2.95 & 3.6 \\
& 640 & $4.1 e-9$ & 2.98 & 3.5 \\
& 1280 & $5.2 e-10$ & 2.98 & 3.5 \\
\hline
\end{tabular}

TABLE 11. DG method with $k=1$ on nonsmooth solution test problems.

\begin{tabular}{ccccc}
\hline Hamiltonian & $1 / \Delta x$ & error & order & $\mathrm{ei}_{h}(u, v)$ \\
\hline convex & 40 & $5.4 e-2$ & - & 4.7 \\
& 80 & $2.8 e-2$ & 0.93 & 4.6 \\
& 160 & $1.4 e-2$ & 0.97 & 4.6 \\
& 320 & $7.2 e-3$ & 0.99 & 4.6 \\
& 640 & $3.6 e-3$ & 0.99 & 6.1 \\
& 1280 & $1.8 e-3$ & 1.00 & 6.1 \\
\hline nonconvex & 40 & $7.5 e-3$ & - & 11 \\
& 80 & $4.6 e-3$ & 0.72 & 9 \\
& 160 & $2.6 e-3$ & 0.82 & 10 \\
& 320 & $1.3 e-3$ & 1.05 & 11 \\
& 640 & $6.7 e-4$ & 0.91 & 16 \\
& 1280 & $3.5 e-4$ & 0.91 & 19 \\
\hline
\end{tabular}

approximation produces smaller errors than the $k=0$ approximation, and that (ii) the $k=0$ approximation failed the parabola test at points close to the kink whereas the $k=1$ approximation did not. Therefore for the $k=1$ approximation, residual computations were performed closer to the kink than for $k=0$. That the resulting effectivity indexes were larger even though the actual error is smaller could be an indication that our a posteriori estimate might be measuring a norm different than the $\mathrm{L}^{\infty}$-norm. This important issue falls beyond the scope of this paper, but will be addressed elsewhere. 
TABle 12. Post-processed DG method with $k=1$ on nonsmooth solution test problems.

\begin{tabular}{ccccc}
\hline Hamiltonian & $1 / \Delta x$ & error & order & $\mathrm{ei}_{h}(u, v)$ \\
\hline convex & 40 & $5.4 e-2$ & - & 4.7 \\
& 80 & $2.8 e-2$ & 0.93 & 4.6 \\
& 160 & $1.4 e-2$ & 0.97 & 4.6 \\
& 320 & $7.5 e-3$ & 0.99 & 4.6 \\
& 640 & $3.6 e-3$ & 0.99 & 6.1 \\
& 1280 & $1.8 e-3$ & 1.00 & 6.1 \\
\hline nonconvex & 40 & $7.5 e-3$ & - & 11 \\
& 80 & $4.6 e-3$ & 0.72 & 9 \\
& 160 & $2.6 e-3$ & 0.82 & 10 \\
& 320 & $1.3 e-3$ & 1.05 & 11 \\
& 640 & $6.7 e-4$ & 0.91 & 16 \\
& 1280 & $3.5 e-4$ & 0.91 & 19 \\
\hline
\end{tabular}

TABLE 13. Post-processed DG method with $k=1$ on nonsmooth solution test problems: The set $[a, b]$ for which the parabola test failed and the optimal value $\epsilon_{\mathrm{opt}}$.

\begin{tabular}{cccccccc}
\hline Hamiltonian & $1 / \Delta x$ & $a$ & $b$ & $|b-a| / \Delta x$ & $\epsilon_{\mathrm{opt}}$ & $\epsilon_{\mathrm{opt}} / E$ & $N \cdot \epsilon_{\mathrm{opt}} / E$ \\
\hline convex & 40 & .431 & .569 & 5.5 & $2.4 e-2$ & 0.18 & 3 \\
& 80 & .466 & .534 & 5.5 & $1.2 e-2$ & 0.16 & 3 \\
& 160 & .483 & .517 & 5.5 & $6.0 e-3$ & 0.14 & 3 \\
& 320 & .491 & .509 & 5.5 & $3.0 e-3$ & 0.12 & 3 \\
& 640 & .494 & .506 & 7.5 & $2.0 e-3$ & 0.14 & 4 \\
& 1280 & .497 & .503 & 7.5 & $9.9 e-4$ & 0.13 & 4 \\
\hline nonconvex & 40 & .469 & .531 & 2.5 & $3.8 e-2$ & 0.25 & 4 \\
& 80 & .484 & .516 & 2.5 & $1.9 e-2$ & 0.22 & 4 \\
& 160 & .489 & .511 & 3.5 & $1.2 e-2$ & 0.23 & 5 \\
& 320 & .495 & .506 & 3.5 & $6.0 e-3$ & 0.21 & 5 \\
& 640 & .496 & .504 & 5.5 & $4.8 e-3$ & 0.30 & 8 \\
& 1280 & .498 & .502 & 5.5 & $2.4 e-3$ & 0.27 & 8 \\
\hline
\end{tabular}

4.5. Two-dimensional experiments. To give an indication that the results obtained in the one-dimensional case can be obtained in the two-dimensional case, we study the behavior of the computational effectivity index for the following monotone

TABLE 14. Two-dimensional test problems.

\begin{tabular}{ccc}
\hline Hamiltonian $H(p)$ & right-hand side $f(x, y)$ & viscosity solution $u(x, y)$ \\
\hline \multirow{2}{*}{$\left.\left|\frac{1}{2}\right| p\right|^{2}$} & $\sin (2 \pi x)+\cos (2 \pi y)$ & $\sin (2 \pi x)+\cos (2 \pi y)$ \\
& $+2 \pi^{2}\left(\cos ^{2}(2 \pi x)+\sin ^{2}(2 \pi y)\right)$ & \\
& $-|\cos (\pi x)|-|\cos (\pi y)|$ & $-|\cos (\pi x)|-|\cos (\pi y)|$ \\
$\frac{1}{\pi^{2}}|p|^{2}$ & $+\sin ^{2}(\pi x)+\sin ^{2}(\pi y)$ & \\
\hline
\end{tabular}


scheme:

$$
\begin{aligned}
v_{i, j}+ & H\left(\frac{v_{i+1, j}-v_{i-1, j}}{2 \Delta x}, \frac{v_{i, j+1}-v_{i, j-1}}{2 \Delta y}\right) \\
& -\omega_{x} \frac{v_{i+1, j}-2 v_{i, j}+v_{i-1, j}}{\Delta x^{2}}-\omega_{y} \frac{v_{i, j+1}-2 v_{i, j}+v_{i, j-1}}{\Delta y^{2}}=f_{i, j},
\end{aligned}
$$

where we can take, for example,

$$
\begin{aligned}
& \omega_{x}=\sup _{(x, y) \in \Omega} \frac{\Delta x}{2}\left|H_{1}\left(\frac{\partial f}{\partial x}(x, y), \frac{\partial f}{\partial y}(x, y)\right)\right|, \\
& \omega_{y}=\sup _{(x, y) \in \Omega} \frac{\Delta y}{2}\left|H_{2}\left(\frac{\partial f}{\partial x}(x, y), \frac{\partial f}{\partial y}(x, y)\right)\right|,
\end{aligned}
$$

and $H_{i}\left(p_{1}, p_{2}\right)=\frac{\partial H}{\partial p_{i}}\left(p_{1}, p_{2}\right)$ for $i=1,2$.

We apply this scheme to the two test problems in Table[14 note that $p=\left(p_{1}, p_{2}\right)$. In our numerical examples, in order to reduce the artificial viscosity of the scheme, we replace $f$ by the exact solution $u$ in the formulae defining $\omega_{x}$ and $\omega_{y}$. Note that the viscosity solution to the second problem is smooth except when either $x=1 / 2$ or $y=1 / 2$.

The implementation of the a posteriori error estimator here is similar to that used for the one-dimensional monotone schemes. We choose $v$ to be the piecewise bilinear interpolant of the numerical solution at the points $\left\{x_{i}, y_{j}\right\}$ and sample at the points $\Omega_{h}=\left\{x_{i+1 / 2}, y_{j+1 / 2}\right\}$. We then have

$$
\begin{aligned}
& v\left(x_{i+1 / 2}, y_{j+1 / 2}\right)=\frac{1}{4}\left(v_{i+1, j+1}+v_{i, j+1}+v_{i+1, j}+v_{i, j}\right), \\
& \frac{\partial v}{\partial x}\left(x_{i+1 / 2}, y_{j+1 / 2}\right)=\frac{1}{2 \Delta x}\left(v_{i+1, j+1}+v_{i+1, j}-v_{i, j+1}-v_{i, j}\right), \\
& \frac{\partial v}{\partial y}\left(x_{i+1 / 2}, y_{j+1 / 2}\right)=\frac{1}{2 \Delta y}\left(v_{i+1, j+1}+v_{i, j+1}-v_{i+1, j}-v_{i, j}\right) .
\end{aligned}
$$

Since there is a well-defined gradient at the points where we are sampling the solution, application of the a posterior error estimator is straightforward.

We take the same range for $\epsilon$ as in the one-dimensional case, namely,

$$
\begin{aligned}
& E=2 \omega|\ln (1 / \omega)|, \\
& N=4|\ln (1 / \omega)|,
\end{aligned}
$$

where now we take $\omega=\max \left\{\omega_{x}, \omega_{y}\right\}$.

In Table 15, we show the results for the smooth solution test problem. We see that the effectivity index remains small and constant as the grid is refined. Since the optimal value of the auxiliary parameter $\epsilon$ is 0 , as expected, no points failed the paraboloid test. 
TABLE 15. Monotone scheme on smooth solution test problem.

\begin{tabular}{ccccc}
\hline $1 \Delta x$ & error & order & $\mathrm{ei}_{h}(u, v)$ & \% of points that failed the paraboloid test \\
\hline 40 & $6.0 e+0$ & 0.94 & 2.2 & 0 \\
80 & $3.0 e+0$ & 0.97 & 2.5 & 0 \\
160 & $1.5 e+0$ & 0.99 & 2.5 & 0 \\
320 & $7.7 e-1$ & 0.99 & 2.6 & 0 \\
\hline
\end{tabular}

TABLE 16. Monotone scheme on nonsmooth solution test problem.

\begin{tabular}{cccc}
\hline $1 / \Delta x$ & error & order & $\mathrm{ei}_{h}(u, v)$ \\
\hline 40 & $1.5 e-1$ & 0.99 & 1.7 \\
80 & $7.8 e-2$ & 1.00 & 1.7 \\
160 & $3.9 e-2$ & 1.00 & 1.8 \\
320 & $2.0 e-3$ & 1.00 & 1.8 \\
\hline
\end{tabular}

TABLE 17. The optimal $\epsilon$ for the monotone scheme on the nonsmooth solution test problem.

\begin{tabular}{ccccc}
\hline $1 / \Delta x$ & $\epsilon_{\mathrm{opt}}$ & $\epsilon_{\mathrm{opt}} / E$ & $N * \epsilon_{\mathrm{opt}} / E$ & $\%$ of points that failed the paraboloid test \\
\hline 40 & $1.6 e-2$ & 0.21 & 4 & 19 \\
80 & $8.0 e-3$ & 0.18 & 4 & 10 \\
160 & $4.0 e-3$ & 0.16 & 4 & 5 \\
320 & $2.0 e-3$ & 0.14 & 4 & 2 \\
\hline
\end{tabular}

Tables 16 and 17 show that the a posteriori error estimate works well in the nonsmooth case. Note, in particular, in Table 17 how the percentage of the grid which fails the paraboloid test is approximately halved with the grid spacing.

In Figure 4, we have plotted the viscosity solution to the nonsmooth test problem on an $80 \times 80$ grid, the absolute value of the error and the region which failed the paraboloid test. Note the rapid variation in the error in the region of the kinks, corresponding to the singularity of the exact solution. Note also how it corresponds well to the singularities in the true solution.

These preliminary results indicate that it is reasonable to expect that in several dimensions the a posteriori error estimate will behave in a manner similar to that observed in one dimension.

4.6. Conclusions. The numerical experiments show that our a posteriori error estimate produces effectivity indexes that remain reasonably constant (and relatively small) as the discretization parameters vary in several orders of magnitude. This is the case not only for the classical monotone scheme but also for the modern finite element high-resolution DG method of $\mathrm{Hu}$ and Shu [10]. This shows that the a posteriori error estimate can be effectively used for quite different types of numerical schemes even in the difficult cases of nonlinear Hamiltonians when the viscosity solution displays kinks. 

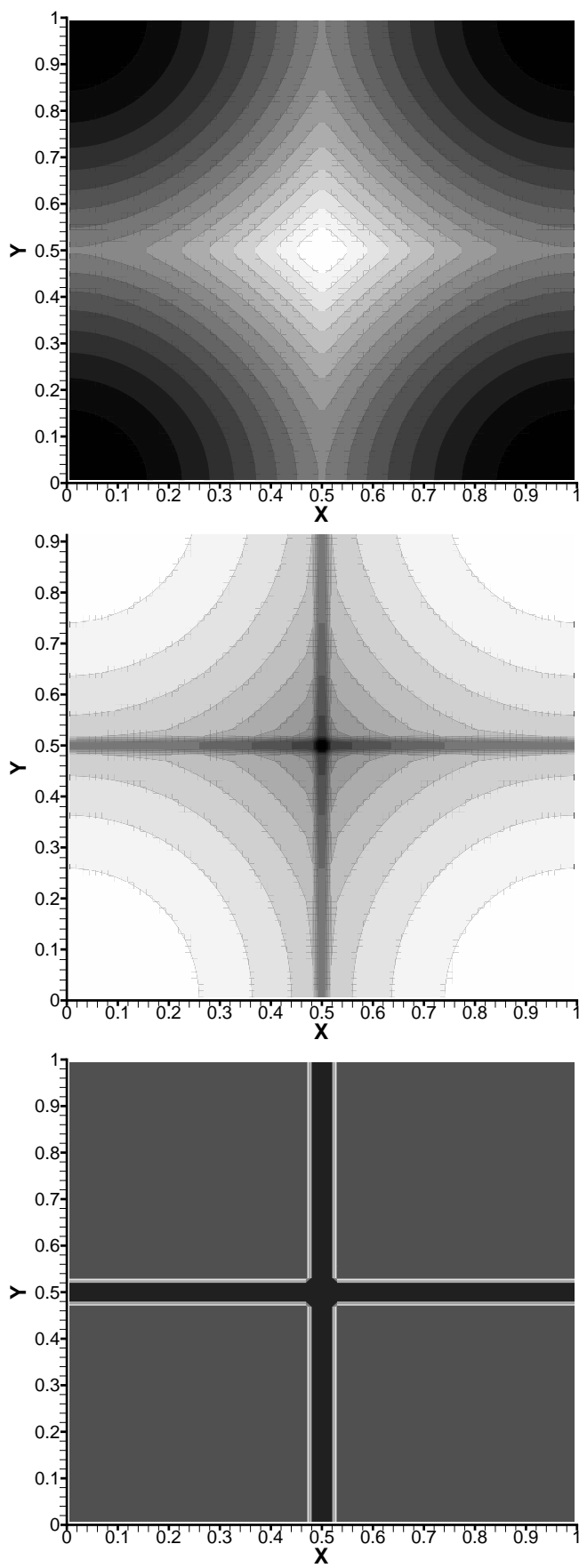

FiguRE 4. Nonsmooth solution test problem, monotone scheme on the $80 \times 80$ grid. Exact solution (top), error (middle), and set that failed the paraboloid test (bottom). Note that this set forms a four-element-wide neighborhood around the kinks of the exact viscosity solution. 


\section{EXTENSIONS AND CONCLUDING REMARKS}

Extension of our a posteriori error estimate to more general Hamiltonians, such as $H=H(x, u, p)$, is straightforward and will not be further discussed. The extension of this result to the transient case and to second-order nonlinear parabolic equations will be treated in the forthcoming Parts II and III, respectively, of this series.

Finally, the technique we have used here on Hamilton-Jacobi equations can be easily carried over to nonlinear hyperbolic scalar conservation laws and, more generally, to nonlinear convection-diffusion scalar equations with possible degenerate diffusion. These subjects will also be considered in forthcoming papers.

Acknowledgments. The authors would like to thank one of the referees, whose criticisms led to a complete revision of the paper, and also Timothy Barth for pointing out mistakes in Table 14 and in the choice of the parameter $\omega$ for the computation of Tables 15 to 17 in an earlier version of the paper.

\section{REFERENCES}

1. R. Abgrall, Numerical discretization of the first-order Hamilton-Jacobi equation on triangular meshes, Comm. Pure Appl. Math. 49 (1996), 1339-1373. MR 98d:65121

2. T. Barth and J. Sethian, Numerical schemes for the Hamilton-Jacobi and level set equations on triangulated domains, J. Comput. Phys. 145 (1998), 1-40. MR 99d:65277

3. B. Cockburn, Discontinuous Galerkin methods for convection-dominated problems, HighOrder Methods for Computational Physics (T. Barth and H. Deconink, eds.), Lecture Notes in Computational Science and Engineering, vol. 9, Springer Verlag, 1999, pp. 69-224. MR 2000f:76095

4. B. Cockburn and H. Gau, A posteriori error estimates for general numerical methods for scalar conservation laws, Mat. Aplic. Comp. 14 (1995), 37-45. CMP 95:15

5. M.G. Crandall, L.C. Evans, and P.L. Lions, Some properties of viscosity solutions of Hamilton-Jacobi equations, Trans. Amer. Math. Soc. 282 (1984), 478-502. MR 86a:35031

6. M.G. Crandall and P.L. Lions, Viscosity solutions of Hamilton-Jacobi equations, Trans. Amer. Math. Soc. 277 (1983), 1-42. MR 85g:35029

7. - Two approximations of solutions of Hamilton-Jacobi equations, Math. Comp. 43 (1984), 1-19. MR 86j:65121

8. K. Eriksson, D. Estep, P. Hansbo, and C. Johnson, Computational Differential Equations, Cambridge University Press, 1996. MR 97m:65006

9. M. Falcone and R. Ferretti, Discrete time high-order schemes for viscosity solutions of Hamilton-Jacobi-Bellman equations, Numer. Math. 67 (1994), 315-344. MR 95d:49045

10. C. Hu and C.-W. Shu, A discontinuous Galerkin finite element method for Hamilton-Jacobi equations, SIAM J. Sci. Comput. 21 (1999), 666-690. MR 2000g:65095

11. G. Kossioris, Ch. Makridakis, and P.E. Souganidis, Finite volume schemes for HamiltonJacobi equations, Numer. Math. 83 (1999), 427-442. MR 2000g:65096

12. C.-T. Lin and E. Tadmor, $L^{1}$-stability and error estimates for approximate Hamiliton-Jacobi solutions, Numer. Math. 87 (2001), 701-735. CMP 2001:09

13. P.L. Lions, E. Rouy, and A. Tourin, Shape-from-shading, viscosity solutions and edges, Numer. Math. 64 (1993), 323-353. MR 94b:65156

14. S. Osher and C.-W. Shu, High-order essentially nonoscillatory schemes for Hamilton-Jacobi equations, SIAM J. Numer. Anal. 28 (1991), 907-922. MR 92e:65118

15. B. Perthame and R. Sanders, The Neumann problem for nonlinear second-order singular perturbation problems, SIAM J. Numer. Anal. 19 (1988), 295-311. MR 89d:35012

16. J.A. Sethian, Level set methods: Evolving interfaces in geometry, fluid mechanics, computer vision, and materials science, Cambridge University Press, Cambridge, 1996. MR 97k:65022

17. P.E. Souganidis, Approximation schemes for viscosity solutions of Hamilton-Jacobi equations, J. Diff. Eqns. 59 (1985), 1-43. MR 86k:35020

18. L.B. Wahlbin, Superconvergence in Galerkin finite element methods, Lecture Notes in Mathematics, vol. 1605, Springer Verlag, 1995. MR 98j:65083 
School of Mathematics, University of Minnesota, 206 Church Street S.E., MinneapoLis, Minnesota 55455

E-mail address: albert@math.umn.edu

School of Mathematics, University of Minnesota, 206 Church Street S.E., MinneapoLis, Minnesota 55455

E-mail address: cockburn@math.umn.edu

Department of Mathematical Sciences, University of Cincinnati, PO Box 210025, Cincinnati, Ohio 45221

E-mail address: french@math.uc.edu

Department of Mathematical Sciences, George Mason University, MS 3F2, Fairfax, VIRGINIA 22030

E-mail address: tpeters1@gmu.edu 